\title{
A Technique for Removal of Forearm Dynamic Compression Plate with Stripped Screws: A Report of Three Cases
}

\author{
CK Lee, MBBS, MK Kwan, MS Ortho, YP Chua, MS Ortho \\ Department of Orthopaedic Surgery, University Malaya Medical Centre, Kuala Lumpur, Malaysia
}

\begin{abstract}
Removal of plates is a procedure commonly performed by orthopaedic surgeons and stripped screws are probably the most common problem encountered during this procedure. Stripped screws are caused by slippage between the screwdriver and the screw. Due to the inherent difficulty in removing such screws, surgeons should be knowledgeable in techniques for their removal and should be equipped with the proper instruments to expedite the procedure. There are few published articles about such techniques. This report describes a technique for removal of plates with stripped screws. The tip of a stripped screw is approached from the far cortex and then reamed with a trephine reamer in the direction of the screw until both cortices are cleared. The plate is then removed with stripped screws attached. All the removals utilizing this technique to date have been successful with no complications, and this method is safe, efficient and technically easy to learn.
\end{abstract}

Key Words:

Stripped screws, Technique, Plate removal, Dynamic Compression Plate

\section{INTRODUCTION}

Fracture fixation with dynamic compression plate (DCP) is an established treatment especially for adult forearm diaphyseal fractures. Removal of this implant is also a common procedure ${ }^{1}$. Some surgeons routinely remove implants once the fracture has united ${ }^{2}$. Sometimes the operating surgeons may encounter difficulties in removing the hardware, and this is most often caused by stripped screws ${ }^{1,3}$ where there is slippage between the screwdriver and the deformed hexagonal-head screw head.

Devices available for removing stripped hexagonal head screws include the screw head gripping vise grip and the conical reverse-cutting male-threaded tap. They are usually made available in the "Screw Removal Set" manufactured by Synthes ${ }^{\circledR}$. There are however circumstances where both of these devices fail. We would like to report an alternative technique for the removal stripped screws used for 3 patients in our centre.

\section{CASE REPORT}

All three patients were male, aged between 16 to 20 years old at the time of injury. Two of the patients sustained closed fracture of the forearm while the other patient had an open forearm fracture. In two patients, both the radius and ulnar bones were fractured while in the third patient only the ulnar was fractured. One of them also sustained closed fracture of the ipsilateral humerus. All fractures were fixed with DCP and hexagonal head cortical screws. There were no complications of the operation and the fractures united well.

These patients presented to our centre for removal of plates 37 to 60 months after fracture fixation. Two were relatively asymptomatic, while one complained of discomfort at the fixation site. All the implants were removed at the Ambulatory Surgery Centre of our institution. At least two stripped screws were encountered in each of the two ulnar DCPs, while the other one was a radial DCP with single stripped screw surrounded by consolidated bone over the edge of the plate. All were removed successfully with this technique without any complications.

\section{Surgical Technique}

When double stripped screw are encountered during removal of a plate, the tip of the stripped screw is approached from the far cortex. A small stab incision was made over the site of far cortex if it cannot be accessed by the old incision wound (Figure 1). The screw tip was identified. A trephine reamer of the appropriate size is placed over the tip of the screw and reamed in the direction of the screw till both far and near cortices are freed from the surrounding bone (Figure 2). This method is repeated for the remaining stripped screw(s). If the stripped screw tip is not visible at the far cortex, the likely location can be found with the help of image intensifying. The DCP is then lifted with gentle manipulation and removed with the stripped screws attached (Figure 3).

We did not perform any bone grafting during the surgery but their forearms were splinted with plaster slab. None of the patients developed refracture of the operated bones. 


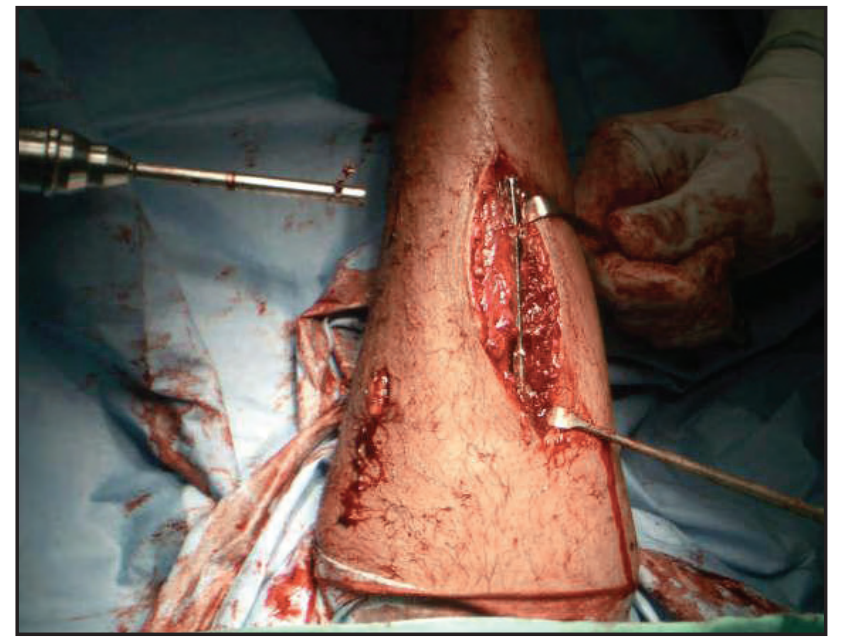

Fig. 1: Stab incision made over the dorsal surface of the forearm and the trephine reamer used to approach the far cortex of the stripped screw.

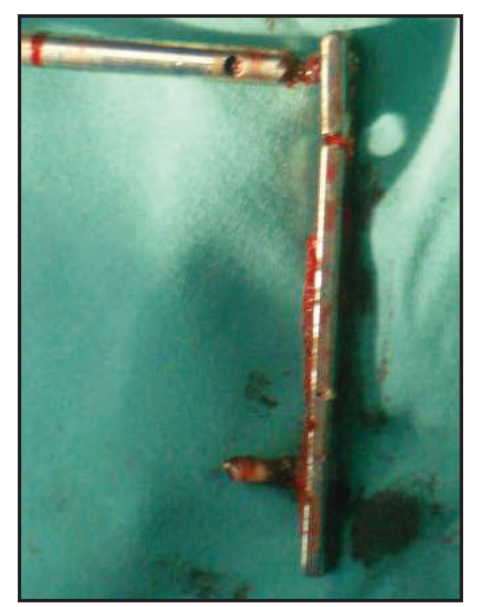

Fig. 3: The plate was removed with the stripped screws still attached to the plate. The trephine reamer of the appropriate size is used.

\section{DISCUSSION}

There are occasions when conventional screw removal devices are not successful in the removal of DCPs with stripped screws. In these three cases, the screws were still strongly fixed to the bone. There was no place to grip the screw using the screw head gripping vise grip as the screw heads were not exposed. As these were small DCPs $(3.5 \mathrm{~mm})$, the screw heads were too small for the reverse-cutting malethreaded tap. The use of this device may also further damage the screw head without providing a good grip on the screw. Therefore, drilling from the far cortex using a trephine reamer is a good alternative in these cases. For cases with a single stripped screw, one can try to loosen the screw by

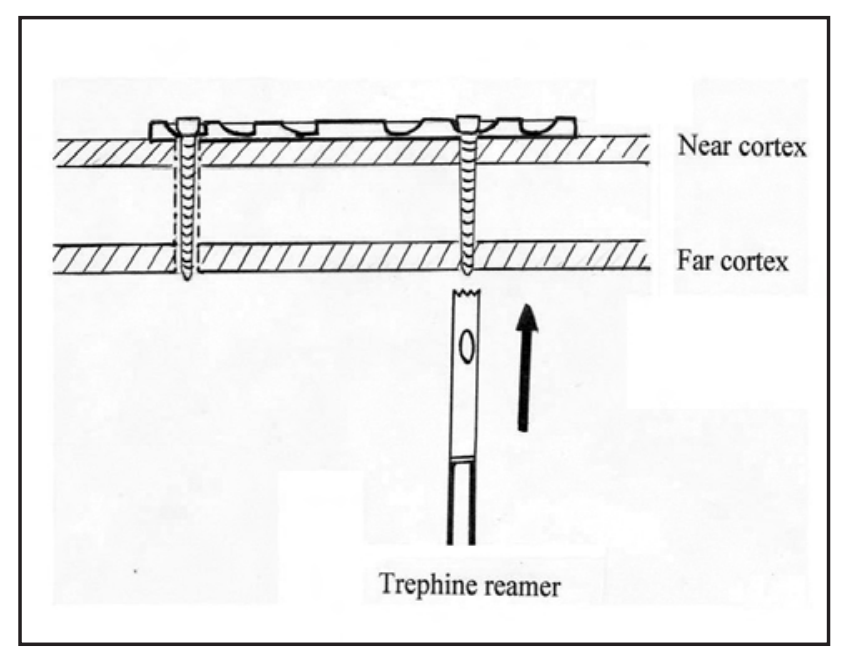

Fig. 2: A drawing diagram of the technique. Note the direction of the trephine reamer from the far cortex.

rotating the plate around the screw. However, if there is a large amount of callus surrounding the plate, this would not be possible.

Locking compression plates have recently gained popularity because the implant comes with the appropriate surgical instrument for this minimally invasive surgery. Due to the locking mechanism of the screw head to the plate and the malleable property of the titanium implant, there is a risk of cold-welding between the screw head and the plate. The removal technique described herein may also prove to be helpful in these situations.

Refracture is the most common complication following removal of implant ${ }^{4,5}$. After removal of plates with stripped screws using this technique, there will be significant risk of refracture considering the larger diameter of bone defect over the reamed site. We suggest cancellous bone grafting should be considered if the resulting defect is relatively big especially for the smaller ulnar bone. The forearm should also be protected from heavy loads by splinting for two to four weeks.

From our case series, we concluded that this technique is safe, efficient and technically easy to learn. All surgeons performing removal of implant should be knowledgeable in dealing with difficulties of hardware removal especially stripped screws. They should have sterile "screw removal set" standby. They should avoid using screwdriver which has already worn off to reduce the incidence of slippage over the hexagonal screw head. This technique should only be indicated in cases with two or more stripped screws or a single stripped screw where the plate is fixed by the excessive surrounding bone." 


\section{REFERENCES}

1. Stafford P, Norris B, Nowotarski P. Hardware removal: tips \& techniques in revision fracture surgery. Techniques in Orthopaedics 2003; 17(4): 522-30.

2. Scales J, Winter G, Shirley H. Corrosion of orthopaedic implants: screws, plates and femoral nail-plates. J Bone Joint Surg 1959; 41-B: 810-20.

3. Behring J, Gjerdet N, Molster A. Slippage between screwdriver and bone screw. Clin Orthop Relat Res 2002; (404): 368-72.

4. Langkamer V, Ackroyd C. Removal of forearm plates - a review of the complications. J Bone Joint Surg. 1990; 72-B: 601-4.

5. Beaupre G, Csongradi J. Refracture risk after plate removal in the forearm. J Orthop Trauma 1996; 10: 87-92. 